\title{
High-intensity lasers: interactions with atoms, molecules and clusters
}

\author{
By M. H. R. Hutchinson ${ }^{2}$, T. Ditmire ${ }^{1}$, E. Springate ${ }^{1}$, \\ J. W. G. TISCh${ }^{1}$, Y. L. ShAO ${ }^{1}$, M. B. MASON${ }^{1}$, \\ N. HAY ${ }^{1}$ AND J. P. MARANGOS ${ }^{1}$ \\ ${ }^{1}$ The Blackett Laboratory, Prince Consort Road, Imperial College of Science, \\ Technology, and Medicine, London SW7 2BZ, UK \\ ${ }^{2}$ Central Laser Facility, Rutherford Appleton Laboratory, \\ Chilton, Didcot OX11 0QX, UK
}

The development of high-intensity short-pulse lasers by chirped pulse amplification is described and the use of short intense pulses for the study of atoms and molecules is discussed. As a case study, the dynamics of the ionization and dissociation of clusters of noble gas atoms is reviewed with special emphasis on the generation of energetic electrons and highly charged ions.

Keywords: clusters; high-intensity lasers; ion energy spectra; electron energy spectra

\section{Introduction}

One of the most significant developments in laser physics over the past decade has been the development of laboratory-scale table-top lasers which are capable of generating optical pulses with powers of over one terawatt. Pulses from such lasers, when focused to small focal spot sizes, can give rise to intensities exceeding $10^{18} \mathrm{~W} \mathrm{~cm}^{-2}$ and can produce novel and unforeseen effects in atoms, molecules and solid materials.

The reason for this development has been the direct result of the technique of chirped pulse amplification (CPA, see Perry \& Mourou 1994). This technique enables two conditions to be met simultaneously. The first is that the energy per unit area or fluence of the pulses should be sufficient to extract efficiently the stored energy from the amplifying medium. This means that the fluence should approach the saturation value for the optical transition which is given by $F_{\text {sat }}=h \nu / \sigma$, where $\sigma$ is the stimulated emission cross-section for the laser transition. For the neodymium:glass laser which operates at a wavelength of $1.05 \mu \mathrm{m}$, the saturation fluence is approximately $6 \mathrm{~J} \mathrm{~cm}^{-2}$ and that for Ti:sapphire $(\lambda \sim 800 \mathrm{~nm})$ is around $1 \mathrm{~J} \mathrm{~cm}^{-2}$. The second requirement is that the pulse duration $(\tau)$ should be as short as possible to maximize the peak power. This is limited by the amplifying bandwidth of the laser medium. For neodymium:glass, the minimum pulse duration is several hundred femtoseconds while that for Ti:sapphire can be very much shorter (Sibbett, this volume). However, for solid state materials, these two criteria cannot be met simultaneously. This is due to the fact the intensity $F_{\text {sat }} / \tau\left(\mathrm{W} \mathrm{cm}^{-2}\right)$ is sufficiently high for the index of refraction of optical materials of the laser to be changed by the radiation. The intensity-dependent refractive index $(n)$ for a pulse of intensity $I$ is given by

$$
n=n_{0}+n_{2} I,
$$

Phil. Trans. R. Soc. Lond. A (1998) 356, 297-315

Printed in Great Britain 
which, for a medium of length $L$, leads to an intensity phase change of

$$
B=\int_{0}^{L} \frac{2 \pi}{\lambda} n_{2} I \mathrm{~d} x
$$

This produces a distortion of the wavefront and, in severe cases, produces selffocusing of the pulse within the amplifying medium and irreversible damage. A practical limit is that $B$ should be less than a few radians and hence this places a limit on the intensity of the pulse which can be propagated in a laser system. A pulse of a given energy can only be amplified in a laser system if the intensity is limited either by increasing the aperture of the optical components or by increasing the pulse duration. The conventional architecture for high-power laser systems involved expanding the beam using laser amplifiers of large apertures, and refocusing the beam at the target to create high intensities. However, this results in inefficient extraction of energy (since $F \ll F_{\text {sat }}$ ) and an increase in scale. The alternative is to lengthen the pulse and keep the aperture sufficiently small for efficient energy extraction $\left(F \sim F_{\text {sat }}\right)$ provided a method can be found to recompress the pulse in time after amplification.

The technique of chirped pulse amplification provides a way of achieving the latter. A short pulse is generated in a mode-locked laser oscillator, stretched in time by factors of several thousand, amplified in conventional laser amplifier, recompressed ideally back on to a target to their original duration and then focused if required. The layout of such a CPA laser is shown in figure 1. Pulses from a mode-locked laser are passed into a pulse stretcher comprising two diffraction gratings and auxiliary focusing optics. This system has negative group velocity dispersion, i.e. the lower frequency components of the pulse follow an optical path which is shorter than that of the higher frequencies and as a result, exit the system first. The output pulse is therefore stretched in time and (positively) frequency chirped so that the instantaneous frequency increases approximately linearly with time. The intensity of the stretched pulse may therefore be reduced by several orders of magnitude. After amplification the reverse process can be performed, again using diffraction gratings but without the focusing optics. By matching the degree of negative group velocity dispersion to the positive dispersion of the stretcher, the original pulse duration may in principle be recovered. However, in practical systems there are several effects which limit the final shape and duration of the compressed pulse. A significant error arises from the fact that the stretcher contains additional focusing optics compared to the compressor. These lenses or mirrors will give rise to various form of aberrations (spherical, chromatic, off-axis phase corrections, etc.) and, particularly in the case of the very short pulses such as are available from mode-locked Ti:sapphire lasers, it is not possible in general to match exactly the group velocity dispersions of the stretcher and compressor over the large bandwidths of the short pulses. This is illustrated in figure 2 which shows the temporal profile of a pulse with an initial duration of $50 \mathrm{fs}$ which had been stretched in stretchers of different designs with lenses, spherical and parabolic mirrors as focusing elements, and then compressed in a conventional two-grating pulse compressor. The effect of uncompensated group velocity dispersion is to generate low-intensity wings or pedestals. Although these can be reduced in intensity to a value which is several orders of magnitude smaller than that of the peak of the pulse, when they are present in a pulse which is focused to very high intensities, $c a .10^{18} \mathrm{~W} \mathrm{~cm}^{-2}$, even a pedestal of $1: 10^{6}$ is sufficient to preionize targets so that the high-intensity peak of the pulse is screened from the target surface

Phil. Trans. R. Soc. Lond. A (1998) 


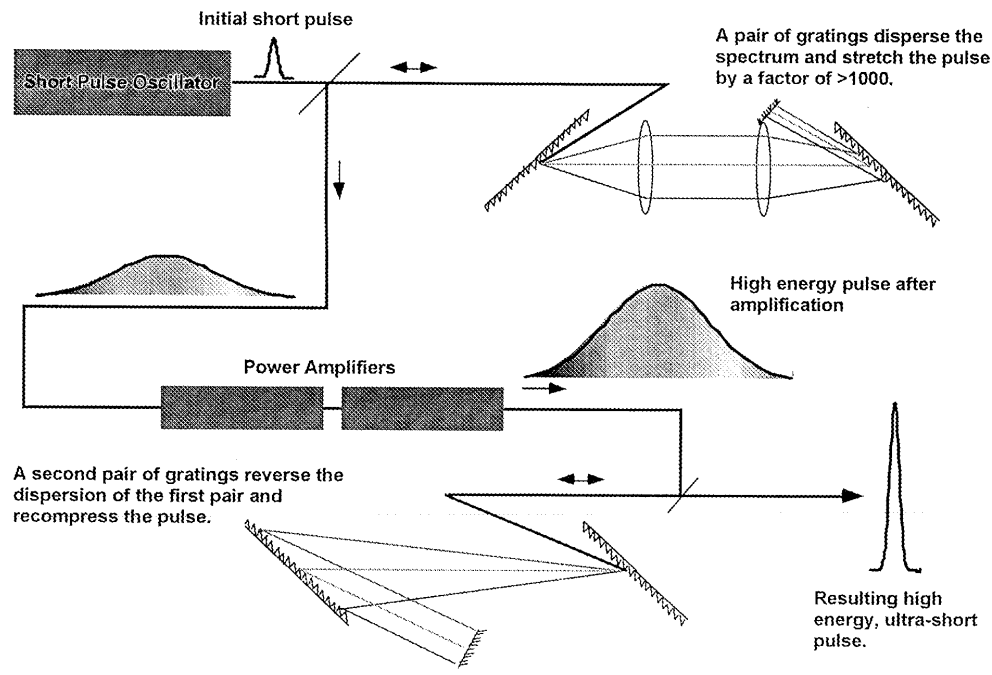

Figure 1. Layout of a CPA laser.

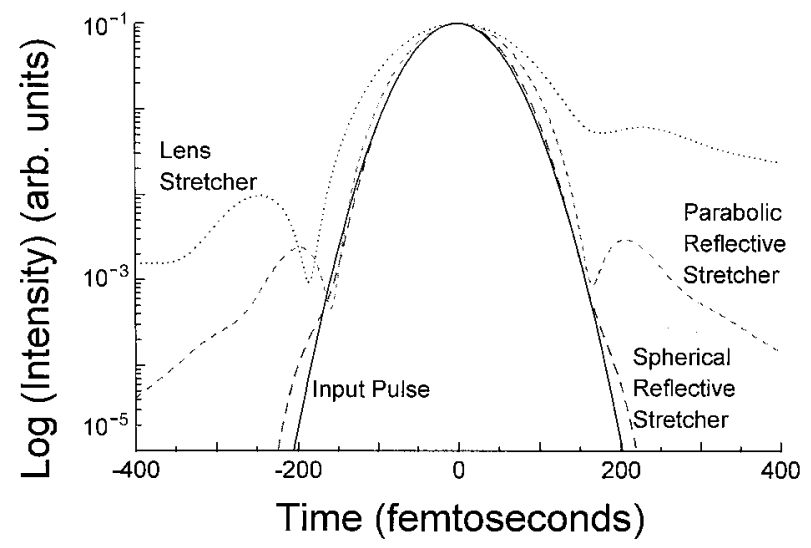

Figure 2. Calculated temporal profile of compressed pulses showing the effect of different designs of pulse stretcher.

by preformed plasma. In addition, dispersion in the various optical components of the laser system will introduce frequency-dependent phase delays on the pulse which causes additional errors. Considerable care must therefore be exercised in the detailed design of stretchers if pulses of less than 50 fs are to be produced. Furthermore, the spectrum of the pulse is narrowed as the pulse is amplified since those frequencies near the peak of the transition lineshape will experience most gain and as a result, the overall bandwidth of the amplified pulse will be reduced. The small frequency bandwidth of the pulses will inevitably result in longer transform-limited pulses when compressed.

The CPA technique has been applied very successfully to solid-state lasers, especially Nd:glass and Ti:sapphire. In both cases, table-top lasers producing powers of terawatts have been produced enabling focused intensities of up to $10^{18} \mathrm{~W} \mathrm{~cm}^{-2}$ to be produced. The CPA technique has also been applied to already existing large-scale Nd:glass lasers increasing the power to 10-100 TW (Walsh et al. 1995; Perry, personal communication). A 1.3 petawatt laser has recently been constructed at Lawrence Livermore National Laboratories. In these cases, the ultimate power is limited by the 


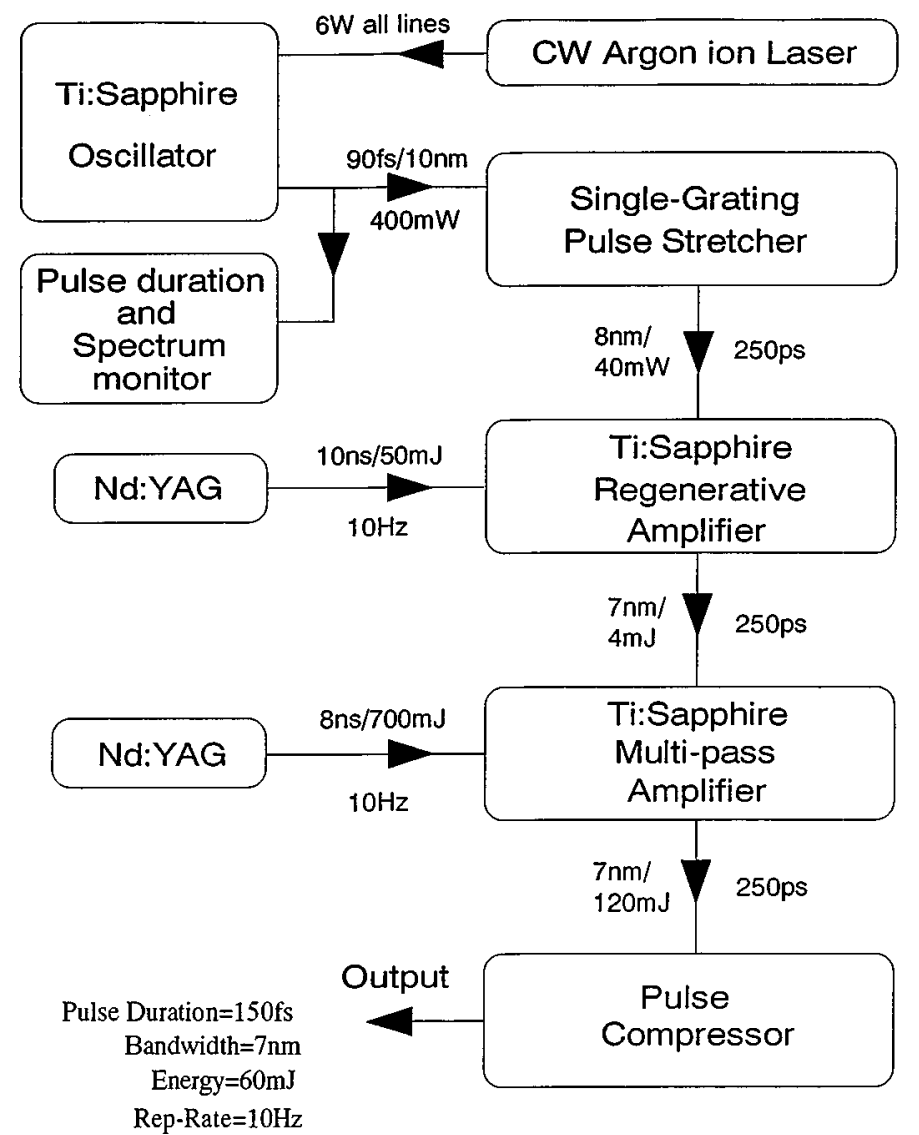

Figure 3. Schematic diagram of Ti:sapphire CPA laser.

availablity of large-aperture diffraction gratings which are capable of withstanding high laser fluences without damage. A very significant development however is the application of the CPA technique to the Ti:sapphire lasers which have very broad bandwidths and are therefore capable of generating very short pulses. These systems may be operated as relatively small-scale table-top systems at high repetition rates using Nd:YAG lasers for pumping the amplifiers. Laser systems with peak powers of up to $25 \mathrm{TW}$ operating at $10 \mathrm{~Hz}$ (Fraser \& Hutchinson 1996; Chambaret et al. 1996) and lower power (1 mJ, $20 \mathrm{fs}$ ) at $1 \mathrm{KHz}$ (Backus et al. 1995) have been constructed and used for applications in atomic, molecular and plasma physics. The layout of a $10 \mathrm{~Hz}$, Ti:sapphire CPA laser system (Fraser \& Hutchinson 1996) is shown in figure 3. This can produce pulses of approximately $140 \mathrm{fs}$ duration with energies of $60 \mathrm{~mJ}$ which can be focused to intensities exceeding $10^{17} \mathrm{~W} \mathrm{~cm}^{-2}$.

\section{Interactions with atoms}

The interaction of laser radiation with atoms is conventionally treated using perturbation theory and provides an adequate description provided the electric field of the radiation is sufficiently small. A useful point of comparison is the electric field experienced by the electron in the ground state of the hydrogen atom and this is 
given by

$$
E_{\text {at }}=e / 4 \pi \varepsilon_{0} a_{0}^{2}
$$

where $a_{0}$ is the Bohr radius. The electric field, $E$, due to radiation and the intensity, $I$, are related by

$$
I=\frac{1}{2} \varepsilon_{0} c E^{2} .
$$

The optical and atomic fields become equal $\left(E=E_{\text {at }}\right)$ for $I=3.6 \times 10^{16} \mathrm{~W} \mathrm{~cm}^{-2}$, an intensity which is reached relatively easily by small-scale CPA lasers. Thus one can expect to encounter physical effects which are not observed at lower intensities where the interaction with atoms is essentially perturbative. (In fact, non-perturbative effects in most atoms are seen at intensities very much less than this value.)

The most prominent interaction channel for atoms at laser intensities above $c a$. $10^{13} \mathrm{~W} \mathrm{~cm}^{-2}$ is above threshold ionization (ATI) in which multiphoton ionization takes place by the absorption of a number of photons which is greater than the minimum required to ionize the atom. Photoelectrons are ejected with discrete energies corresponding to multiple photon absorption. In such cases, the energy of the photoelectrons produced by ATI are relatively low, typically less than $100 \mathrm{eV}$. At higher laser intensities ( $c a .10^{15} \mathrm{~W} \mathrm{~cm}^{-2}$ ) the predominant ionization mechanism is by tunnelling through the Coulomb potential which is distorted by the applied electric field. Multiples of the frequency of the incident radiation are also produced and, although harmonic generation at lower intensities under perturbative conditions is well known, it is found that, with increasing intensity, the efficiency of harmonic generation no longer decreases monotonically with increasing order but that a plateau is reached where a wide range of harmonic frequencies are produced with similar intensities. High harmonic generation can be described very successfully by a semiclassical treatment in which the active electron which is driven by the oscillating electric field collides with the atomic core and radiates. The maximum energy $\left(E_{\max }\right)$ of the resulting radiation is related to the maximum kinetic energy the electron can acquire in the field. Thus

$$
E_{\max }=I_{\mathrm{p}}+3.2 U_{\mathrm{p}}
$$

where $I_{\mathrm{p}}$ is the ionization potential of the atom and $U_{\mathrm{p}}=e^{2} E^{2} / 4 m \omega^{2}$ is the pondermotive energy of the electrons, i.e. the time-averaged energy of oscillation in the electromagnetic field with electric field amplitude $E$ and angular frequency $\omega$. $\left(U_{\mathrm{p}}=9.3 \times 10^{-14} I \lambda^{2} \mathrm{eV}\right.$, where $I$ is the intensity in $\mathrm{W} \mathrm{cm}^{-2}$ and the wavelength $\lambda$ is in $\mu \mathrm{m}$.) For the helium atom, $I_{\mathrm{p}}=24.6 \mathrm{eV}$ so that, for a Nd:glass laser $(\lambda=1.05 \mu \mathrm{m})$ at an intensity of $3 \times 10^{14} \mathrm{~W} \mathrm{~cm}^{-2}$, the wavelength of the harmonic with the maximum energy is $10.6 \mathrm{~nm}$ corresponding to a harmonic order of 99. Using rare gases as the atomic species, harmonics of orders as high as 143 (corresponding to a wavelength of $7.4 \mathrm{~nm}$ for the Nd:glass laser; see Perrry \& Mourou (1994)) have been produced and, using the UV KrF laser, 37th harmonic generation at $6.7 \mathrm{~nm}$ has been observed (Preston et al. 1996).

\section{Interaction with plasmas}

The plasmas produced by the intense illumination of solids at these high intensities have also been investigated. Unlike single atoms, intense laser interactions with highdensity plasmas can be very energetic. These plasmas efficiently absorb laser light due to rapid inverse bremsstrahlung heating of the plasma electrons (Murnane et al.

Phil. Trans. R. Soc. Lond. A (1998) 
1991). Such plasmas can exhibit average electron temperatures of up to $1 \mathrm{keV}$, though they are usually clamped to lower values because of conductive cooling of the plasma. The expansion of hot laser-heated plasmas is usually followed by a hydrodynamic expansion into vacuum, resulting in the ejection of fast ions.

\section{Interaction with molecules}

The interaction of a short pulse of intense laser radiation with a simple diatomic molecule leads to multiphoton ionization by mechanisms very similar to those of single atoms. If electrons are removed from both constituent atoms the resulting repulsive electrostatic force leads to a Coulomb explosion (Codling \& Frasinski 1994) in which the molecule dissociates yielding ionic fragments with energies which are determined in part by the internuclear separation when ionization took place. In addition, if the laser field is sufficiently strong so as to distort the potentials of the molecule, the dissociation dynamics of the molecule may be altered, giving rise to bond softening (Zavriyev et al. 1990), above-threshold dissociation (Guisti-Suzor et al. 1990) and the creation of light-induced vibrational states (Zavriyev et al. 1993). Although the dynamics of Coulomb explosions of triatomic molecules has been studied successfully, in general the detailed study of such processes becomes increasingly difficult as the size of the molecules increases.

\section{Interaction with atomic clusters}

Atomic clusters have been studied by chemists and physicists because of the unique position that clusters hold as an intermediate state between molecules and solids (Castleman \& Keesee 1988). Many studies have traced the properties of materials from their monatomic characteristics to their bulk-state characteristics through an examination of the material as it forms larger and larger clusters. Photofragmentation studies in particular have been very useful in illuminating the chemistry of these atomic and molecular clusters. Many experiments involving single-photon and multiphoton ionization of clusters with long laser pulses (ca. nanoseconds) at low to moderate intensities have been performed, and the photofragmentation (Chen et al. 1990; Nagata et al. 1991) and Coulomb explosion (Snodgrass et al. 1989; Li \& Whetten 1992) dynamics have been extensively investigated.

Recently, there has been intense activity in extending these studies to very-highintensity ultrashort laser pulses with peak laser intensities higher than $10^{15} \mathrm{~W} \mathrm{~cm}^{-2}$ and pulse widths of 0.1-10 ps (McPherson et al. 1994; Purnell et al. 1994; Ditmire et al. 1996). In this regime the physics governing the interaction is fundamentally different than in previous studies since, at these intensities, the laser interaction is non-perturbative and very-high-order multiphoton ionization and strong electricfield tunnel ionization is possible. Consequently, highly charged ions can be produced (McPherson et al. 1994; Shao et al. 1996; Ditmire et al. 1997a,b). Since the short pulses used are comparable to, or shorter than, the disassembly times of a cluster in the laser field (Ditmire et al. 1996), the entire laser pulse interacts with an inertially confined body of atoms.

The dramatic difference between the nature of intense laser interactions with atoms and solid density plasmas raises an interesting question. Do clusters of a few hundred to a few thousand atoms which are small compared to a laser wavelength behave like smaller molecules in strong fields or more like the energetic plasmas produced from

Phil. Trans. R. Soc. Lond. A (1998) 


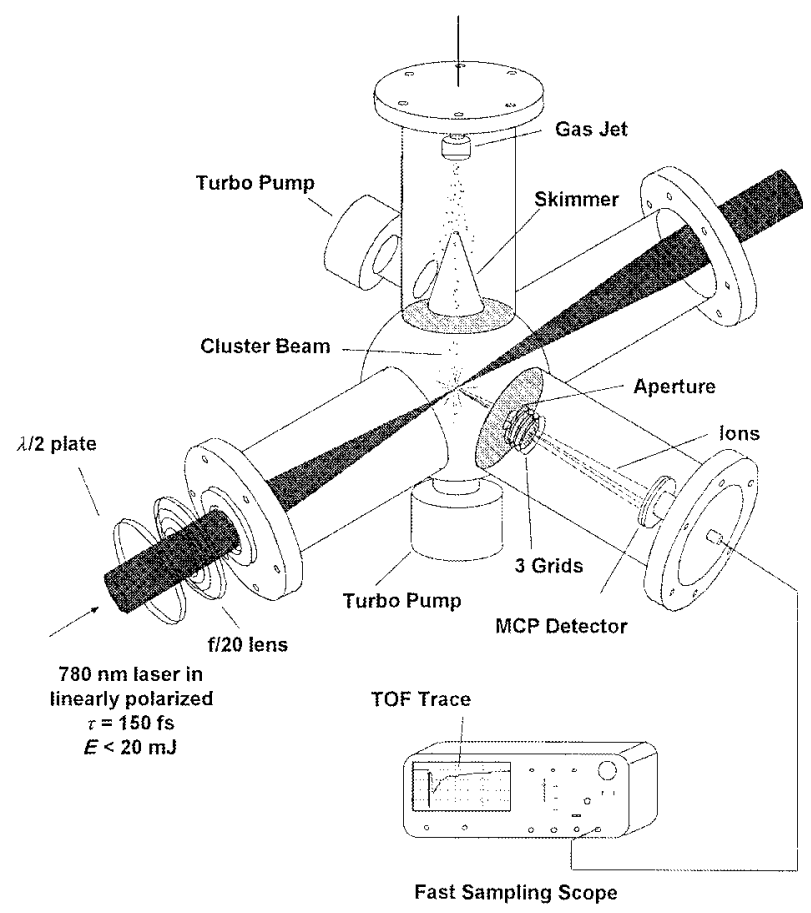

Figure 4. Experimental configuration for measuring ion energies of exploding clusters.

solids? Initial studies of a variety of cluster sizes using lasers of wavelengths between $248 \mathrm{~nm}$ and $1054 \mathrm{~nm}$ indicated that the intense laser interaction with clusters was much more energetic than interactions at similar intensities with atoms or small molecules. These initial studies found that intense irradiation of a medium of clusters resulted in very intense X-ray emission. In fact, X-rays with energies up to $5 \mathrm{keV}$ were observed in a Xe cluster gas jet irradiated by $248 \mathrm{~nm}$ pulses at intensities higher than $10^{18} \mathrm{~W} \mathrm{~cm}^{-2}$ (McPherson et al. 1994). Similar high X-ray yields have been observed in cluster media of various species and at a range of laser wavelengths (Chen et al. 1990). These studies indirectly indicated that the clusters were absorbing substantial fractions of laser energy and were producing hot electrons and high-charged ions capable of producing the observed radiation.

Clusters of the heavy noble gases, xenon, krypton and argon form clusters when the gas is cooled by adiabatic expansion through a nozzle and are thus convenient systems for experimental study. To study their dissociation, a beam of atomic clusters, produced in the expansion of a high-pressure gas into vacuum, is irradiated by a focused high-intensity femtosecond laser beam as shown in figure 4 . The electrons and ions expelled from the clusters with velocities perpendicular to both the cluster beam and the laser beam propagate along a time-of-flight (TOF) tube and are detected by a micro-channel plate detector (MCP). The ion energies may then be determined by measurement of their flight time; the electron energies are found by measuring the decrease in the MCP signal when a retarding voltage is applied to grids placed between the laser focus and the MCP.

The laser used is a high-power Ti:sapphire CPA laser which delivers $40 \mathrm{~mJ}$ pulses of $150 \mathrm{fs}$ duration at a wavelength of $780 \mathrm{~nm}$ (Fraser \& Hutchinson 1996). This laser is focused to yield a peak focused intensity of approximately $2 \times 10^{16} \mathrm{~W} \mathrm{~cm}^{-2}$ with $20 \mathrm{~mJ}$ of laser intensity. The noble gas clusters are produced in a jet by a pulsed 


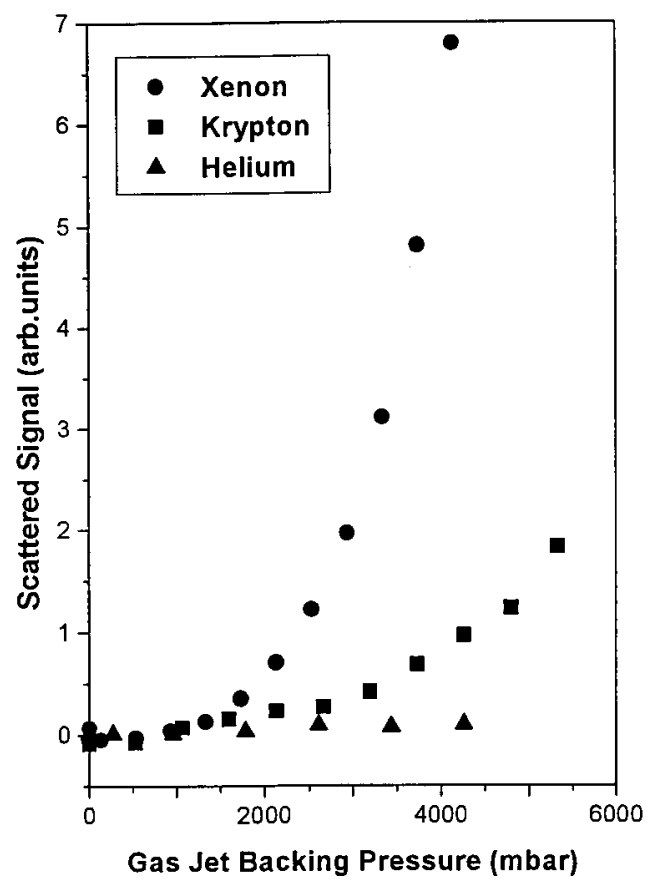

Figure 5. Measured Rayleigh scattered light signal as a function of gas-jet backing pressure for xenon, krypton and helium.

solenoid gas valve. Using the scaling laws determined by Hagena \& Obert (1972), the average number of atoms in the clusters at the onset of condensation of the gas can be estimated from the dimensions of the jet, the gas and the backing pressure.

The presence of clusters in the gas jet and a determination of their average size may be obtained from Rayleigh scattering measurements. Low-power vertically polarized $532 \mathrm{~nm}$ light from a frequency-doubled Nd:YAG laser is focused into the gas jet and the side-scattered light from this region detected. The scattered signal as a function of backing pressure, $p_{0}$, for xenon, krypton and helium is shown in figure 5 . These measurements indicate that clusters of a detectable size begin to form at pressures around 1000 mbar in xenon and 2000 mbar in krypton. Helium is not expected to cluster under these conditions and no scattered signal above the noise level is observed. The scattered signal from the Xe and $\mathrm{Kr}$ clusters displays a $p_{0}^{3}$ dependence with increasing backing pressure. This is consistent with simple scaling arguments from which it is possible to estimate the cluster size as a function of the gas pressure. It may be inferred that, at $p_{0}=4 \mathrm{bar}$, an average xenon cluster will contain approximately 1600 atoms and have a radius $R_{\mathrm{c}} \approx 46 \pm 10 \AA$, while a krypton cluster will typically contain approximately 500 atoms and have a radius $R_{\mathrm{c}} \approx 28 \pm 7 \AA$.

\section{Electron energy spectra}

The measured energy spectrum of electrons emitted along the direction of the laser polarization during the irradiation of clusters of approximately 2100 xenon atoms (4.5 bar backing pressure) with an intensity of $1.5 \times 10^{16} \mathrm{~W} \mathrm{~cm}^{-2}$ is shown in figure 6 . There are two distinct features in the electron energy spectrum. The first, broad, peak consists of so-called 'warm electrons' with energies ranging from 0.1 to 


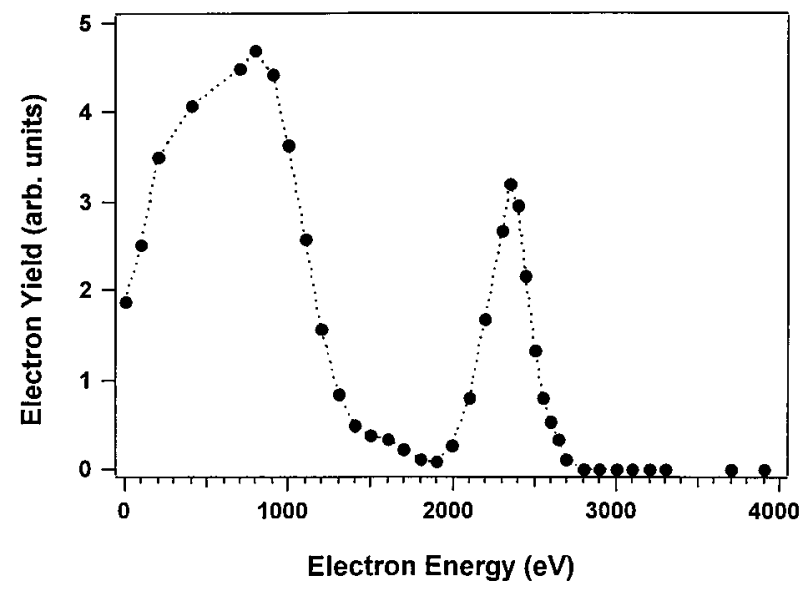

Figure 6. Measured electron energy distribution from xenon clusters for a peak intensity of $1.5 \times 10^{16} \mathrm{~W} \mathrm{~cm}^{-2}$. The gas-jet backing pressure was $4.5 \mathrm{bar}$, corresponding to an average cluster size of $50 \AA$ or 2100 atoms.

$1 \mathrm{keV}$. A second, sharper, peak (referred to here as the 'hot electrons') appears at $2.5 \mathrm{keV}$. Both peaks are only present when the laser interacts with Xe clusters. When the interaction region contains only monatomic xenon (in a static fill), no electrons with energy above $100 \mathrm{eV}$ are detected.

The most remarkable aspect of this energy distribution are the high electron kinetic energies, with a large fraction of the electrons having energies between 2 and $3 \mathrm{keV}$. Previous measurements of ATI spectra from single atoms at this intensity and pulse duration have indicated that the vast majority of electrons produced have energies below $100 \mathrm{eV}$ (Walker et al. 1994). Only a very small fraction of electrons (typically $10^{-3}-10^{-4}$ ) have higher energy, with no detectable electrons having an energy of above $1 \mathrm{keV}$. The spectrum observed from Xe clusters clearly indicates a much greater coupling of laser energy to electrons than is present during the irradiation of single atoms. Furthermore, this spectrum indicates that the laser-cluster interaction produces even hotter electron temperatures than a laser-solid interaction at this intensity, where electron temperatures of $100-500 \mathrm{eV}$ are typical (Shepherd et al. 1994).

The yield of hot electrons as a function of gas-jet backing pressure provides more evidence that this signal results from the interaction of the laser with clusters rather than atoms. There is close correlation in the pressure at the onset of hot-electron production with the onset of clustering in xenon; both hot-electron production and clustering have a sharp onset with a gas-jet backing pressure of 1 bar. The presence of two distinct peaks in the electron energy spectrum suggests that these two groups may be produced under different conditions at different times in the cluster expansion. This assumption is supported by examining the angular dependence of the electron emission with respect to the laser polarization (figure 7). The angular distributions of the two groups of electrons are markedly different. The hot-electron emission is completely isotropic while warm-electron emission is peaked along the laser polarization with a width of about $60^{\circ}$. Both these distributions are significantly different from the angular distributions associated with single atoms; electrons from high-order ATI normally have a much narrower angular distribution. In highfield tunnelling ionization, the narrow angular distribution arises from the much higher tunnelling rate in the direction of the laser field. The electrons observed from

Phil. Trans. R. Soc. Lond. A (1998) 

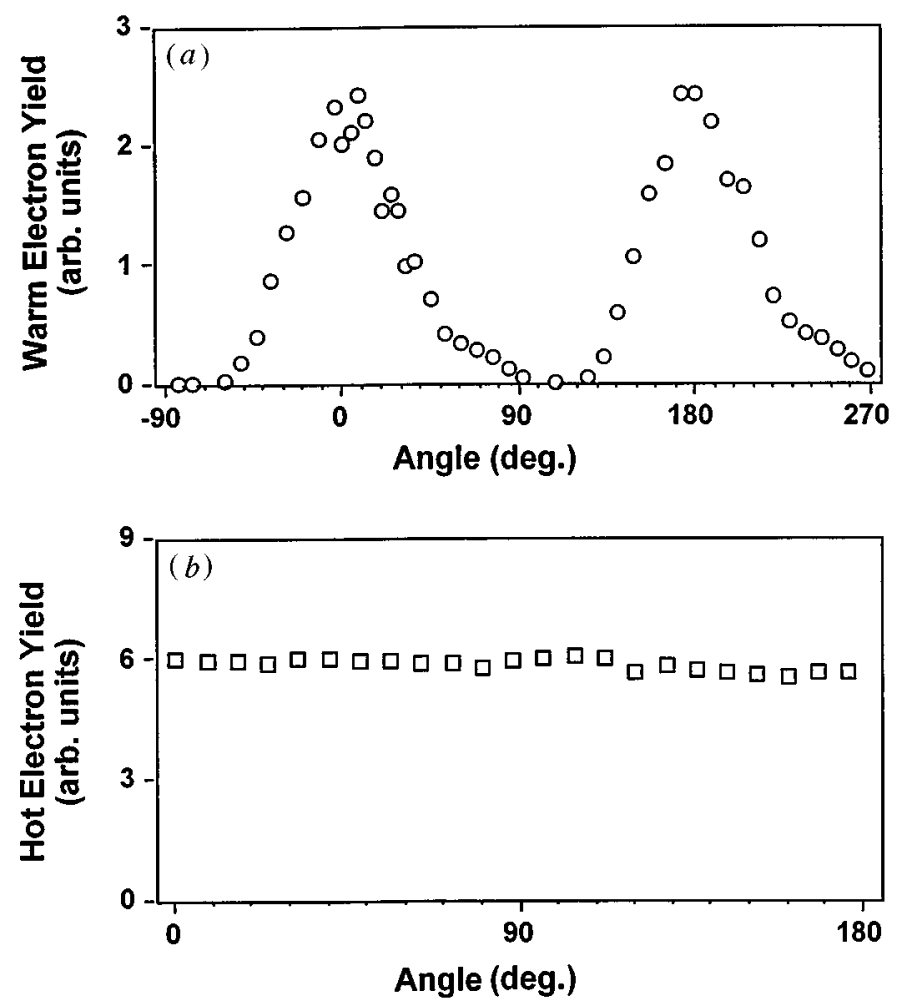

Figure 7. (a) Angular distribution of 'warm electrons' with energies ranging from 0.3 to $1 \mathrm{keV}$. Emission along the direction of laser electric-field polarization is defined as being at $0^{\circ}$ and $180^{\circ}$. (b) Angular distribution of 'hot electrons' with energies ranging from 2 to $3 \mathrm{keV}$. The gas-jet backing pressure was 4 bar, corresponding to an average cluster size of 1600 atoms.

clusters cannot therefore be interpreted as simply resulting from the tunnel ionization of individual atoms. Some rescattering of the electrons by ions in the cluster is necessary to explain the broadening observed in the warm-electron distribution. The isotropic distribution of the hot electrons indicates that these electrons have undergone many electron-ion collisions in the laser field, completely randomizing their velocity distribution.

\section{Ion energy spectra}

The remarkably high energies of the electrons produced in the intense laser-cluster interaction suggests that highly charged ions with large kinetic energies may also be ejected from the cluster. Charge separation of these hot electrons will inevitably accelerate the cluster ions to high velocities.

The energy spectrum of ions resulting from the interaction of 2500-atom Xe clusters with a laser pulse of intensity around $2 \times 10^{16} \mathrm{~W} \mathrm{~cm}^{-2}$ is shown in figure 8 . The most remarkable aspect of this energy distribution is the presence of ions with energies up to $1 \mathrm{MeV}$. This energy is four orders of magnitude higher than has previously been observed in the Coulomb explosion of molecules (Augst et al. 1991) and about 1000 times higher than the energy of Ar ions ejected in the disintegration of small clusters of up to six argon atoms irradiated at $10^{15} \mathrm{~W} \mathrm{~cm}^{-2}$ reported by Purnell et 


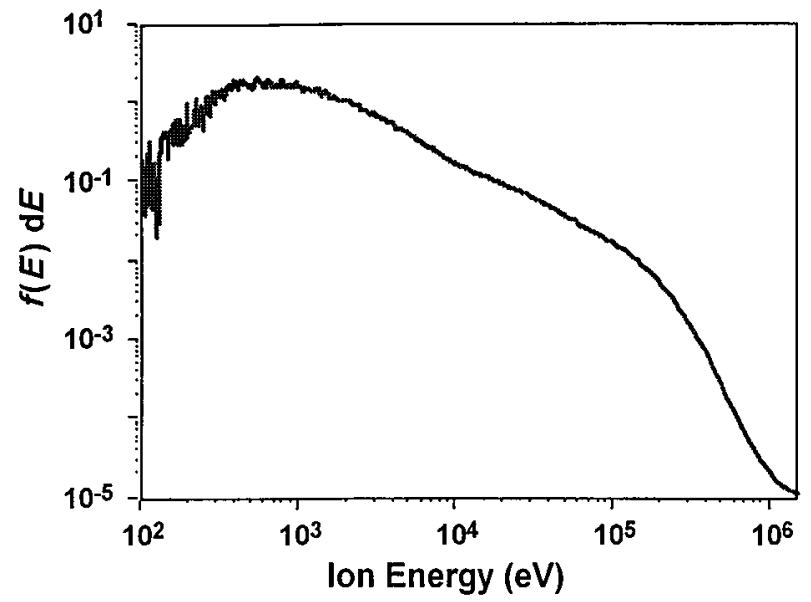

Figure 8. Ion energy spectrum from clusters of 2500 Xe atoms (backing pressure 5 bar) irradiated by a peak intensity of $2 \times 10^{16} \mathrm{~W} \mathrm{~cm}^{-2}$, derived from the time-of-flight trace shown in figure 3 .

al. (1994). The average ion energy of this distribution, defined as

$$
\bar{E}=\frac{\int E f(E) \mathrm{d} E}{\int f(E) \mathrm{d} E},
$$

is $45 \pm 5 \mathrm{keV}$. Thus the average laser energy deposited per ion is also substantial. In addition it is found that the ion energy distribution is isotropic with respect to the direction of laser polarization, apparently a consequence of a spherically exploding cluster.

The ability to control the cluster size by changing the backing pressure of the gas jet allows examination of the scaling of the ion energy distribution with cluster size. However, there is no dramatic shift in the shape of the ion distribution as the cluster size is increased, suggesting that the mechanism driving the cluster ion explosion does not dramatically change as the cluster size is varied from a few hundred to a few thousand atoms per cluster. This general behaviour is also found in the explosion of $\mathrm{Kr}$ clusters, though the explosions are not as energetic as those of the Xe clusters under similar conditions.

\section{Charge state distributions}

A striking feature of the ions produced from the cluster explosion is ionization to very-high-charge states, a feature that has already been noted by other authors (McPherson et al. 1994; Ditmire et al. 1995). Figure 9 shows the time-of-flight spectra from around 2500-atom xenon clusters irradiated by $2 \times 10^{16} \mathrm{~W} \mathrm{~cm}^{-2}$ at kinetic energies from $1-100 \mathrm{keV}$ as the retarding voltage, $\Phi$, is varied from $0-3.3 \mathrm{kV}$. (The retarding voltage introduces a potential barrier to ions with energies less than $Z e \Phi$, without significantly altering the flight times of higher energy ions.) The charge state distributions calculated from these spectra are shown in figure 10.

For high-energy Xe ions, the peak is at $Z=18^{+}-25^{+}$, with some ions having charge states as high as $40^{+}$. The peak for high-energy krypton ions is at $12^{+}-17^{+}$with the highest charge state present being around $Z=25^{+}$. These are much higher charge

Phil. Trans. R. Soc. Lond. A (1998) 


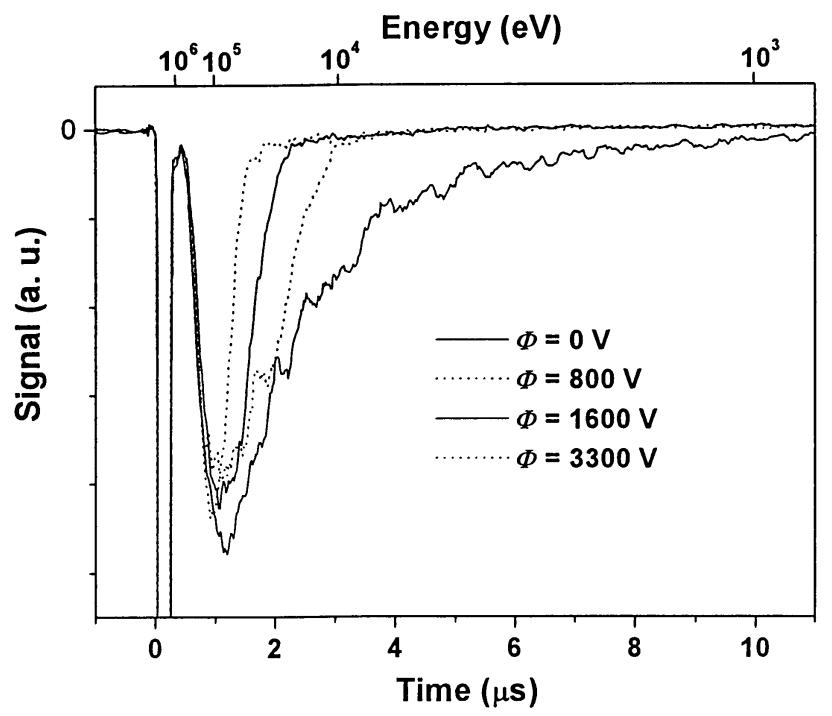

Figure 9. Ion TOF traces with $38 \mathrm{~cm}$ flight tube of 2500 -atom Xe clusters (backing pressure 5 bar) irradiated with a peak intensity of $2 \times 10^{16} \mathrm{~W} \mathrm{~cm}^{-2}$ at retarding voltages of $0,800,1600$ and $3300 \mathrm{~V}$.

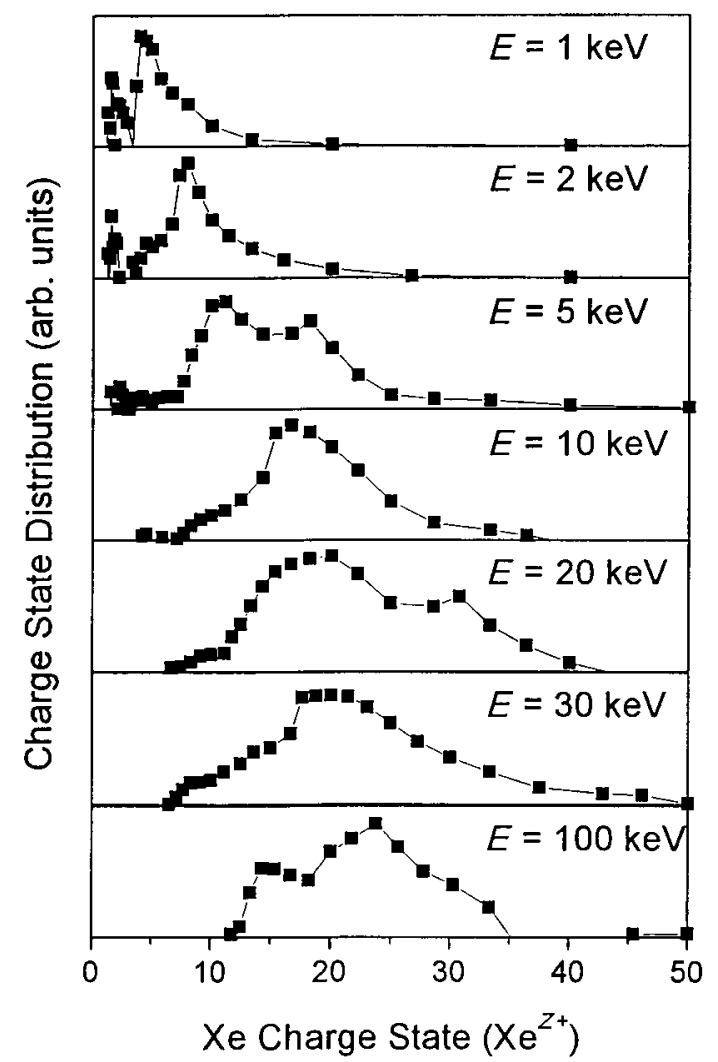

Figure 10. Measured charge state distribution of 2500-atom Xe clusters (backing pressure 5 bar) irradiated with a peak intensity of $2 \times 10^{16} \mathrm{~W} \mathrm{~cm}^{-2}$ for ion kinetic energies of $1,2,5,10,20,30$ and $100 \mathrm{keV}$. 
states than those expected from field ionization of single atoms at these intensities. One would expect to see charge states up to $12^{+}$in xenon and $8^{+}$in krypton at an intensity of $2 \times 10^{16} \mathrm{~W} \mathrm{~cm}^{-2}$ (Ditmire et al. 1995). Ionization to $\mathrm{Xe}^{40+}$ would require an intensity of nearly $10^{20} \mathrm{~W} \mathrm{~cm}^{-2}$ if the ionization were due to tunnel ionization alone. In the cluster, high-temperature electrons, which are created through laserdriven heating, strip the ions to higher charge states by collisional ionization. The ion charge state depends only weakly on ion kinetic energy, contrary to what would be expected from a simple Coulomb explosion and to the results reported for the Coulomb explosion of very small argon clusters (Purnell et al. 1994).

\section{Theoretical and numerical analysis}

The very-high-energy particles observed experimentally are dramatically different then those typically produced in strong field laser interactions with molecules and are more typical of particles produced in the interaction of a high-intensity laser with solid density plasmas. This suggests that the appropriate way to explain the exploding cluster behaviour is to treat it as a small microplasma. Consequently one may treat the ionized cluster as a classical spherical plasma ball of uniform density. This treatment is appropriate when the charge of the cluster is sufficient to retain electrons within the vicinity of the cluster following their ionization from the constituent atoms.

This picture of the cluster implies a number of interesting consequences. First, because of the high electron and ion densities within the cluster, electron collisional processes will be very important. In particular, the electrons will undergo rapid heating by the laser field due to electron-ion collisions (inverse bremsstrahlung). This process converts the coherent oscillation energy of the electron cloud to random thermal energy. Electron collisional ionization will also be important, stripping the constituent atoms to very-high-charge states.

The second consequence of viewing the cluster as a sphere of plasma is that the cluster, which becomes conducting once some electrons have been liberated by ionization, will exhibit some of the optical properties of metallic clusters. In particular, a giant resonance in the optical absorption spectrum (Brechignac \& Connerade 1994) occurs when the light frequency is near to the plasma frequency of the electrons in the cluster and is a result of a resonantly driven oscillation of the entire cluster electron cloud. (The actual plasma density at the resonance is dependent upon the shape of the cluster.) The presence of this resonance is very important in the dynamics of the high-intensity laser interaction with the cluster. The numerical model may be constructed (Ditmire et al. 1996) which treats the cluster as a spherical microplasma, subject to the standard processes of a laser-heated plasma. Ionization in the cluster is by tunnelling and both thermal- and laser-driven electron collisions. The model calculates the free streaming rate of electrons leaving the cluster, accounting for the mean free path of electrons in the cluster. Only electrons with energy sufficient to overcome the Coulomb attraction of the positively charged cluster are allowed to leave. The cluster expansion, assumed to be uniform and isotropic, is calculated accounting for hydrodynamic and Coulomb repulsion forces within the charged cluster and the electron energy distribution within the cluster is assumed to be Maxwellian.

A zero-frequency approximation for the laser field may be used to account for the collective electron oscillation effects on the optical absorption of the cluster. This

Phil. Trans. R. Soc. Lond. A (1998) 
approximation is appropriate when the cluster is much smaller than the laser wavelength and assumes that the response of the cluster electron cloud is fast compared to the time scale of the cluster expansion dynamics. Hence, one can calculate the electric field inside the cluster using the expression for the electric field of a dielectric sphere in a uniform electric field (Jackson 1975). This is

$$
E=E_{0} \frac{3}{|\varepsilon+2|},
$$

where $E_{0}$ is the laser electric field in a vacuum. The cluster dielectric constant is given by the Drude model for a plasma:

$$
\varepsilon=1-\left(\frac{n_{\mathrm{e}}}{n_{\text {crit }}}\right)(1+\mathrm{i} \nu / \omega)^{-1},
$$

where $n_{\mathrm{e}}$ is the electron density, $n_{\text {crit }}$ is the electron critical density for a laser field of frequency $\omega$, and $\nu$ is the electron-ion collision frequency. This formula predicts that when $n_{\mathrm{e}} / n_{\text {crit }}>6$ the electric field inside the cluster is shielded by the oscillating electron cloud and the field inside the cluster is smaller then the surrounding field in vacuum. On the other hand, $E$ has a sharp maximum when $n_{\mathrm{e}} / n_{\text {crit }}=3$. At this point the oscillating laser field resonantly drives the cluster electron cloud and the field inside the cluster is enhanced. As a result, the free electrons in the cluster undergo rapid collisional heating because of the local increase in the field energy density.

An example of the dynamics of a xenon cluster are illustrated in figure 11. Here the calculated time history of a $60 \AA$ Xe cluster (approximately 3600 atoms) irradiated by a $140 \mathrm{fs}$ pulse with a peak intensity of $2 \times 10^{16} \mathrm{~W} \mathrm{~cm}^{-2}$ is shown. The laser pulse envelope is shown in figure $11 a$. Figure $11 b$ illustrates the radius of the cluster as a function of time, figure $11 c$ shows the electron density (normalized to the critical density), and figure $11 d$ shows the electron temperature. From these curves, the general history of the cluster interaction with the laser pulse can be seen.

Early in the rising edge of the laser pulse, around $t \approx-190 \mathrm{fs}$, optical ionization creates some free electrons in the cluster. Though a few of the initially ionized electrons escape from the initially neutral cluster, the space charge of the cluster keeps additional electrons from escaping. These electrons will then start to acquire thermal energy from the laser field through Coulomb collisions with the ions in the cluster. The temperature of the electrons begins to rise at $t \approx-175 \mathrm{fs}$. In addition, the free electrons in the cluster will begin to collisionally ionize the Xe ions in the cluster. The cluster then begins to expand and the electron heating continues. The heating of the electrons in the initial phase of the interaction is low due to the shielding of the laser field by the high free-electron density that has been created in the cluster. Ultimately, at $t \approx-30 \mathrm{fs}$, the expansion of the cluster lowers the electron density to bring the electron oscillation into resonance with the laser field. This results in a very rapid deposition of the energy into the electrons, causing the sharp spike in the electron temperature seen in figure $11 d$. At this point, the ions are stripped very rapidly by the hot electrons. The energy density of the cluster is in excess of $300 \mathrm{~J} \mathrm{~cm}^{-2}$ corresponding to an electron pressure of more than 10 Mbar. The cluster then explodes, manifested by the rapid expansion seen in figure $11 b$ following the resonant heating.

Using these calculations it is possible to track the dynamics of the electrons escaping from the cluster. Figure 12 shows the time history of a $50 \AA$ Xe cluster (approximately 2100 atoms) irradiated by a pulse with a peak intensity of $1 \times 10^{16} \mathrm{~W} \mathrm{~cm}^{-2}$.

Phil. Trans. R. Soc. Lond. A (1998) 

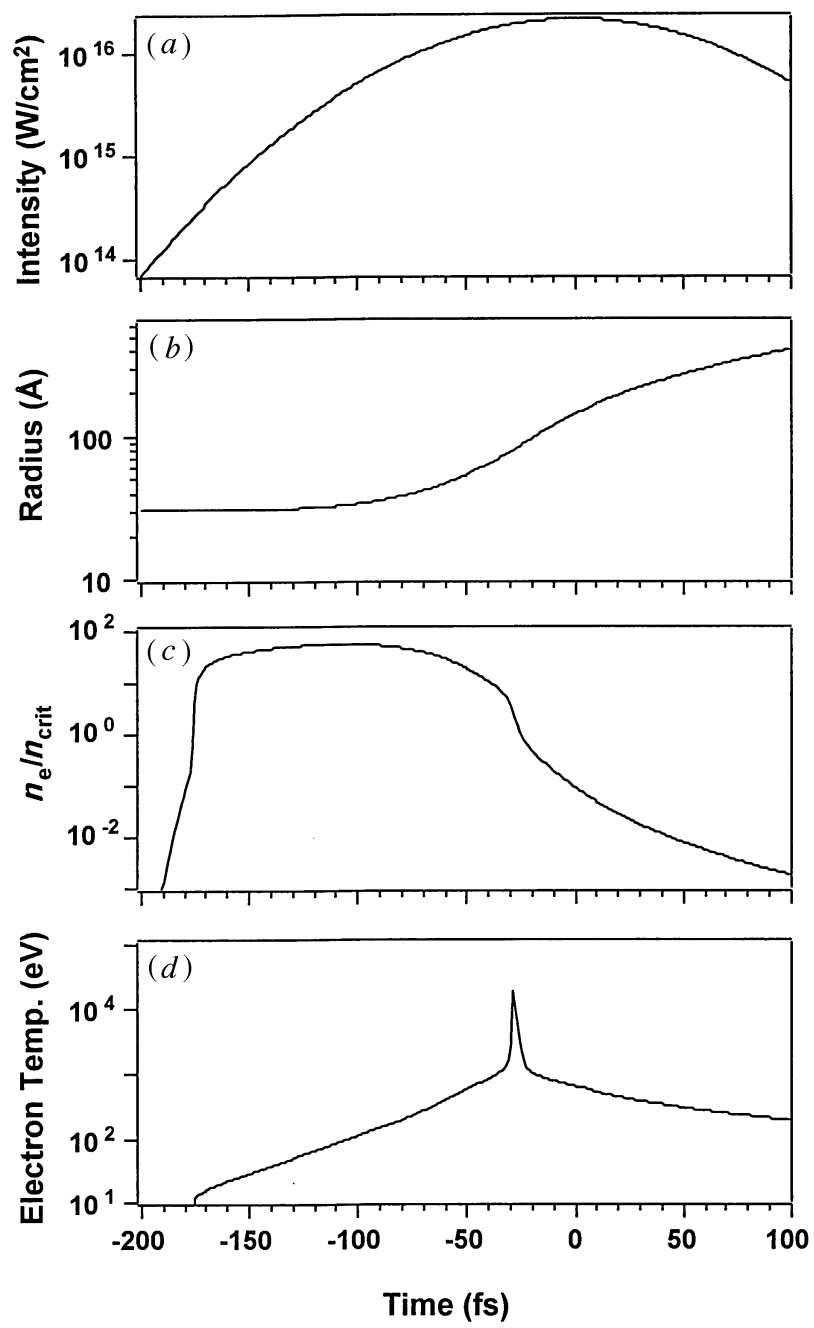

Figure 11. Theoretical calculations of the time history of $60 \AA$ Xe clusters $(3600$ atoms per cluster) irradiated by a 140 fs pulse with a peak intensity of $2 \times 10^{16} \mathrm{~W} \mathrm{~cm}^{-2}$. (a) Laser pulse envelope; $(b)$ cluster radius; $(c)$ electron density, $n_{\mathrm{e}}$ (normalized to the critical density, $n_{\text {crit }}$ ); and $(d)$ electron temperature.

Here the electron temperature, laser-pulse envelope and escape energy threshold are shown, along with the calculated rate at which electrons exit the cluster by free streaming. Some electrons escape from the cluster in the initial stages of the cluster interaction, as the electron temperature rises but, once the electron temperature peaks due to the heating by the giant resonance, the electron escape rate also peaks sharply since many of the electrons acquire enough thermal energy to overcome the space-charge forces of the cluster. This history implies that the electron energy spectrum might exhibit two features, one arising from the lower energy electrons which escape from the cluster early in the interaction and one from the hot electrons that escape during the resonance heating of the cluster, in qualitative agreement with the experimental observations.

The calculated electron distribution for the dynamics described in figure 12 is shown in figure 13. The calculated distribution does, in fact, exhibit a two-lobed 


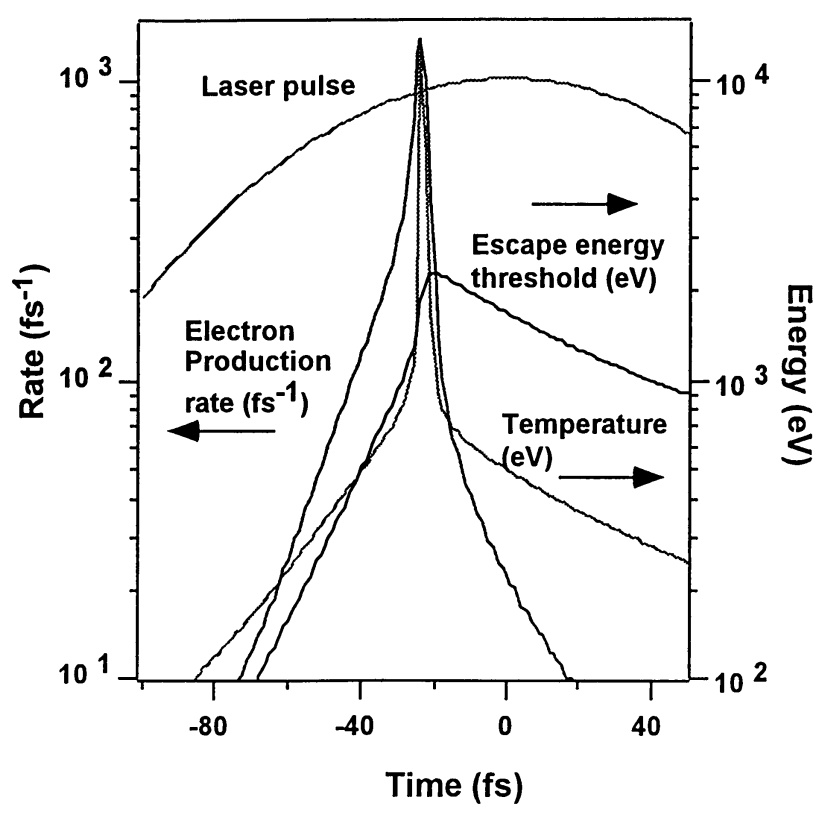

Figure 12. Time history of $50 \AA$ Xe cluster (2100 atoms per cluster) at $1 \times 10^{16} \mathrm{~W} \mathrm{~cm}^{-2}$ showing the laser pulse envelope, the electron temperature, the rate at which electrons exit the cluster by free streaming and the escape energy threshold.

distribution and exhibits a close similarity to the measured electron distribution. The sharp peak near $2.5 \mathrm{keV}$ is clearly consistent with the observed data, both in position and its narrow width. The calculation indicates that this peak in the data is evidence for the giant resonance in the heating of the electrons in the cluster spherical microplasma. The warm electron peak is the result of collisional heating of electrons near the surface of the cluster on the rising edge of the laser pulse. These electrons have undergone a limited number of collisions, broadening the angular distribution from that of purely tunnel-ionized electrons. The hot electrons result from rapid collisional heating of the remaining electrons in the bulk of the cluster later in the pulse when the electron density drops to a point to bring the heating into resonance. Consequently, their velocity distribution has been completely randomized, accounting for the isotropic distribution observed.

The production of hot electrons through inverse bremsstrahlung can drive a very energetic explosion of the cluster and charge separation of the hot electrons will inevitably drive a rapid expansion of the cluster. The explosion of the cluster can be driven by two forces. The first is the Coulomb repulsion between the highly charged ions in the cluster. If all the free electrons are retained in the cluster, the cluster is quasineutral and this force is negligible. However, the free streaming of electrons from the cluster will cause a charge build-up of the plasma sphere, and a Coulomb 'pressure' will develop.

The second force important in driving the cluster explosion is the hydrodynamic pressure of the free electrons in the cluster. This force is present even if the cluster plasma remains neutral. The hot electrons in a plasma will set up a radial ambipolar potential which then accelerates the cluster ions. The pressure driving this expansion mechanism is simply given by

$$
P_{\text {hyd }}=n_{\mathrm{e}} k_{\mathrm{B}} T_{\mathrm{e}}
$$

Phil. Trans. R. Soc. Lond. A (1998) 


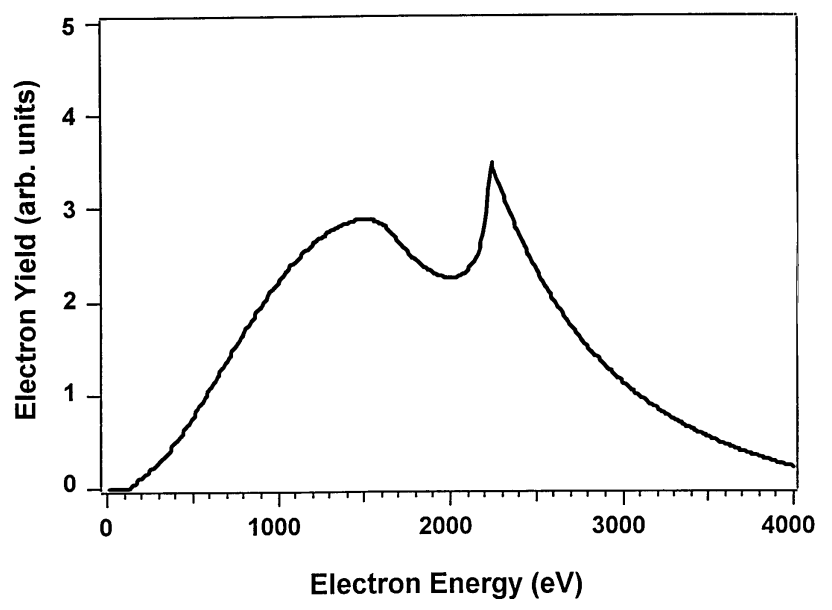

Figure 13. Calculated electron energy spectrum for $50 \AA$ Xe clusters (2100 atoms per cluster) irradiated by a $140 \mathrm{fs}$ pulse with a peak intensity of $2 \times 10^{16} \mathrm{~W} \mathrm{~cm}^{-2}$ (as in figure 12).

where $T_{\mathrm{e}}$ is the electron temperature. This hydrodynamic pressure is the same force that drives the expansion of a solid target plasma into vacuum after it has been heated by an intense laser pulse. In the interaction of picosecond pulses at intensities of higher than $10^{16} \mathrm{~W} \mathrm{~cm}^{-2}$ fast ions resulting from these plasmas have been observed with energies up to a few hundred keV (Meyerhofer et al. 1993).

The Coulomb explosion mechanism is similar to the mechanism that drives the explosion of small optically ionized molecules. However, in bulk solid plasmas, the plasma remains quasineutral and the expansion is driven by the hydrodynamic force. This difference points to the question of which mechanism is responsible for the explosion of the clusters observed in these experiments. The scaling laws of these forces suggests that Coulomb explosion forces may dominate for small clusters whereas the hydrodynamic force dominates for larger systems (more than 1000 atoms). The appearance of the high-ion-charge states observed (greater than $20^{+}$for Xe ions) is predicted due to rapid collisional ionization by the hot electrons within the cluster which can strip the ions to very-high-charge states ( up to $\mathrm{Xe}^{40+}$ ), in agreement with the experimental observations of figure 10 .

\section{Conclusion}

The development of compact high-intensity CPA lasers has allowed novel areas of atomic, molecular and plasma physics to be explored. This has produced new insights into the nature of the interaction of light with matter and has produced sources of coherent short-wavelength radiation by high harmonic generation. The interaction with atomic clusters with more than a few hundred atoms represents an important transition in the dynamics of intense laser-matter interactions from molecules to solids and provides an appropriate case study, giving rise to highly energetic electrons and highly stripped ions, species which could only be produced by conventional lasers of many times their intensity.

This work was supported in part by the UK Engineering and Physical Sciences Research Council and the MOD.

Phil. Trans. R. Soc. Lond. A (1998) 


\section{References}

Augst, S., Meyerhofer, D. D., Strickland, D. \& Chin, S. L. 1991 Laser ionization of noble gases by Coulomb-barrier suppression. J. Opt. Soc. B 8, 858.

Backus, S., Peatross, J., Huang, C. P., Murnane, M. M. \& Kapteyn, H. C. 1995 Ti-Sapphire amplifier producing millijoule-level, $21 \mathrm{fs}$ pulses at $1 \mathrm{KHz}$. Opt. Lett. 20, 2000.

Brechignac, C. \& Connerade, J. P. 1994 The giant resonance in atoms and clusters. J. Phys. B 27, 3795.

Castleman, A. W. \& Keesee, R. G. 1988 Gas-phase clusters: spanning the states of matter. Science 241, 36.

Chambaret, J. P., Leblanc, C., Cheriaux, G., Curley, P., Darpentigny, G., Rousseau, P., Hamoniaux, G., Antonetti, A. \& Salin, F. 1996 Generation of $25 \mathrm{tw}, 32 \mathrm{fs}$ pulses at $10 \mathrm{~Hz}$. Opt. Lett. 21, 1921.

Chen, Z. Y., Cogley, C. D., Hendricks, J. H., May, B. D. \& Castleman, A. W. 1990 Photodissociation of $\mathrm{Kr}_{n}^{+}$clusters. J. Chem. Phys. 93, 3215.

Codling, K. \& Frasinski, L. J. 1994 Coulomb explosion of simple molecules in intense laser fields. Contemp. Phys. 35, 243.

Ditmire, T., Donnelly, T., Falcone, R. W. \& Perry, M. D. 1995 Strong X-ray emission from high temperature plasmas produced by intense irradiation of clusters. Phys. Rev. Lett. 75, 3122.

Ditmire, T., Donnelly, T., Rubenchik, A. M., Falcone, R. W. \& Perry, M. D. 1996 Interaction of intense laser pulses with atomic clusters. Phys. Rev. A 53, 3379.

Ditmire, T., Tisch, J. W. G., Springate, E., Mason, M. B., Hay, N., Marangos, J. \& Hutchinson, M. H. R. 1997a High energy ion explosion of super-heated clusters: transition from molecular to plasma behavior. Phys. Rev. Lett. 78, 2732.

Ditmire, T., Tisch, J. W. G., Springate, E., Mason, M. B., Hay, N., Smith, R. A., Marangos, J. \& Hutchinson, M. H. R. 1997a High-energy ions produced in explosions of superheated atomic clusters. Nature 386, 54 .

Fraser, D. J. \& Hutchinson, M. H. R. 1996 High intensity titanium-doped sapphire laser. J. Mod. Opt. 43, 1055.

Guisti-Suzor, A., He, X., Atabek, O. \& Mies, F. H. 1990 Above-threshold dissociation of $\mathrm{H}_{2}^{+}$in intense laser fields. Phys. Rev. Lett. 64, 515.

Hagena, O. F. \& Obert, W. 1972 Cluster formation in expandion supersonic jets: effect of pressure, temperature, nozzle size, and test gas. J. Chem. Phys. 56, 1793.

Jackson, J. D. 1975 Classical electrodynamics. New York: Wiley.

Li, X. L. \& Whetten, R. L. 1992 Stability islands for doubly charged clusters below the kinetic critical size. Chem. Phys. Lett. 196, 535.

McPherson, A., Thompson, B. D., Borisov, A. B., Boyer, K. \& Rhodes, C. K. 1994 Multiphotoninduced X-ray emission at $4-5 \mathrm{keV}$ from Xe atoms with multiple core vacancies. Nature $\mathbf{3 7 0}$, 631.

Meyerhofer, D. D., Chen, H., Delettrez, J. A., Soom, B., Uchida, S. \& Yaakobi, B. 1993 Resonance absorption in high-intensity contrast, picosecond laser-plasma interactions. Phys. Fluids B 5, 2584.

Murnane, M. M., Kapteyn, H. C., Rosen, M. D. \& Falcone, R. W. 1991 Ultrafast X-ray pulses from laser-produced plasmas. Science 251, 531

Nagata, T., Hirokawa, J. \& Kondo, T. 1991 Photodissociation of $\mathrm{Ar}_{n}^{+}$cluster ions. Chem. Phys. Lett. 176, 526.

Perry, M. D. \& Mourou, G. 1994 Terawatt to petawatt subpicosecond lasers. Science 264, 917.

Preston, S. G., Sanpera, A., Zepf, M., Blyth, W. J., Smith, C. G., Wark, J. S., Key, M. H., Burnett, K., Nakai, M., Neely, D. \& Offenberger, A. A. 1996 High-order harmonics of $248.6 \mathrm{~nm}$ KrF laser from helium and neon ions. Phys. Rev. A 53, R31.

Purnell, J., Snyder, E. M., Wei, S. \& Castleman Jr, A. W. 1994 Ultrafast laser-induced Coulomb explosion of clusters woth high charge states. Chem. Phys. Lett. 229, 333.

Shao, Y. L., Ditmire, T., Tisch, J. W. G., Springate, E., Marangos, J. P. \& Hutchinson, M. H. R. 1996 Multi-keV electron generation in the interaction of intense laser pulses with Xe clusters. Phys. Rev. Lett. 77, 3343.

Phil. Trans. R. Soc. Lond. A (1998) 
Shepherd, R., Price, D., White, W., Gordan, S., Osterheld, A., Walling, R., Goldstein, W. \& Stewart, R. 1994 Characterization of short pulse laser-produced plasmas. J. Quant. Spec. Rad. Trans. 51, 357.

Snodgrass, J. T., Roehl, C. M. \& Bowers, M. T. 1989 Photodissociation dynamics of $\mathrm{Ar}_{3}^{+}$. Chem. Phys. Lett. 159, 10.

Walker, B., Sheehy, B., DiMauro, L. F., Agostini, P., Schafer, K. J. \& Kulander, K. C. 1994 Precision measurement of strong field double ionization of helium. Phys. Rev. Lett. 73, 1227.

Walsh, F. N.,Barr, J. R. M., Barzanti, L. J.,Danson, C. N., Ebbage, M. D., Edwards, C. B., Gander, M. J., Hanna, D. C., Hughes, D. W., Hutchinson, M. H. R., Key, M. H., Majdabadi, A. A., Mercer, I. P., Neely, D., Norreys, P. A., Pepler, D. A., Rivers, S. A., Taday, P. F., Toner, W. T., Winstone, T. B. \& Zhou, F. 1995 Greater than $10^{19} \mathrm{~W} \mathrm{~cm}^{-2}$ irradiances for laser/plasma studies interaction. Institute of Physics Conference Series, vol. 140, pp. 451-454.

Zavriyev, A., Bucksbaum, P. H., Muller, M. G. \& Schumacher, D. W. 1990 Ionization and dissociation of $\mathrm{H}_{2}$ in intense laser fields at $1.064 \mu \mathrm{m}, 532 \mathrm{~nm}$ and $355 \mathrm{~nm}$. Phys. Rev. Lett. 42, 5500 .

Zavriyev, A, Bucksbaum, P. H., Squier, J. \& Saline, F. 1993 Light-induced vibrational structure in $\mathrm{H}_{2}^{+}$and $\mathrm{D}_{2}^{+}$in intense laser fields. Phys. Rev. Lett. 70, 1077. 
